\title{
La calidad de vida en los adultos mayores \\ y su percepción en la calidad de la atención de Enfermería
}

Mtro. Rey Arturo Salcedo Álvarez*, Mtra. Rosa Amarilis Zárate Grajales**, Mtra. María Dolores Zarza Arizmendi***, Mtra. Virginia Reyes Audiffred****, Lic. Severino Rubio Domínguez***** y Mtro. Guillermo Fajardo Ortiz ******.

*Profesor de Riesgos y Daños para la Salud, División de Estudios de Posgrado ENEO; **Jefa de la División de Estudios de Posgrado, ENEO; ***Secretaria General Académica, ENEO; ****Coordinadora de Extensión Académica y Servicios de Enfermería Universitaria ENEO; *****Director de la ENEO y ******Profesor Titular "C" Facultad de Medicina UNAM.

El presente trabajo aborda la importancia del nivel de Calidad de Vida (CV) en los Adultos Mayores (AM) y la percepción de la Calidad de la Atención de Enfermería (CAE). El objetivo fue conocer la percepción de la CAE que tienen los AM de acuerdo a sus características sociodemográficas de salud y a su CV.

Durante junio de 2006 con el apoyo de cuatro académicos y alumnos de la ENEO se encuestó a AM en tres centros de atención médica y en diversos puntos geográficos del D. F.

Resultados: se entrevistaron 82 AM, 52 mujeres y 30 hombres, la calificación que asignaron a la CAE fue considerada como: "Muy Buena" o "Buena" en 80\%, el análisis de correlación entre CV y la CAE mostró baja correlación.

Las conclusiones fueron que en general los AM tienen buena opinión de la CAE; las mujeres tienen una mejor opinión que los hombres, las opiniones desfavorables aumentan conforme se incrementa la edad, el bajo nivel educativo, la pobreza y la severidad del diagnóstico; las opiniones más favorables están relacionadas con la mayor necesidad de cuidado, aparentemente la CV no determina la percepción de la CAE cuando la necesidad de cuidado es mínima.

PALABRAS CLAVE: Calidad de vida, calidad de la atención, Enfermería, adultos mayores.

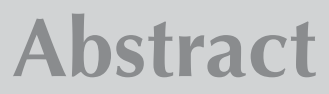

The present work investigates the importance of the quality of life (QL) in major adults (MA) and the perception of the care quality in nursery (QAN). The object was to know the perception of the QAN that the MA have according to their socio-demographic, health and QL characteristics.

During June of 2006 with the support of academicians and students of the ENEO were polled MA in three centres of medical attention in diverse geographical points in Mexico City.

Results: were polled 82 MA, 52 women and 30 men. The assigned qualification to (QAN) was assigned as "very good" or "good" in 80\%, the correlation analysis between the (QL) and the (QAN) showed a low correlation.

Conclusions were, in general that the MA have a good opinion of the (QAN), women have a better opinion of men, the unfavourable opinions are increased as increases the age, the low educative level, the poverty and the severity of the diagnosis, the most favourable opinions are related to the greater necessity of care, apparently the QL does not determine the perception on the QAN when the necessity of care is minimum.

KEY WORDS: Quality of life, care quality, nursing, major adults. 


\section{INTRODUCCIÓN}

La Calidad de la atención es un tema que cada día toma mayor interés entre los profesionales de la salud. La Cruzada Nacional por la Calidad de los Servicios de Salud fue el primer programa con cobertura nacional enfocado a fortalecer la calidad de la atención en salud, este programa se instituyó para dar respuesta al reto de la calidad de la atención'. Garantizar la calidad en salud es un hecho deseable, que se debe de entender como parte de un sistema continuo de retroalimentación en donde la definición de los procedimientos es esencial y necesaria antes de la medición y evaluación. En el caso de Enfermería en México², la "Comisión Interinstitucional de Enfermería" (Hoy Comisión Permanente de Enfermería) del Sistema Nacional de Salud, concretó los "Indicadores de Calidad de la Atención de Enfermería", con la definición, monitoreo y evaluación de éstos indicadores se estarán dando los primeros pasos para la sistematización de la "Auditoria de Salud en Enfermería" y ahora se podrá tener un mejor control del cumplimiento de las normas, se vigilará más eficientemente el uso de los recursos, se detectarán tempranamente las fallas en el cuidado, se podrán hacer oportunamente recomendaciones específicas para mejorar los procedimientos de atención y se permitirá la evaluación constante. La "Comisión Interinstitucional de Enfermería definió la calidad de los servicios de Enfermería como "la atención oportuna, personalizada, humanizada, continua y eficiente que brinda el personal de Enfermería, de acuerdo con estándares definidos para una práctica profesional, competente y responsable, con el propósito de lograr la satisfacción del usuario y del prestador de servicios". 3

En 1998, la Organización Mundial de la Salud presentó su informe anual con base al análisis de las tendencias demográficas y de salud desde 1955 y proyectadas al año 2025; como resultado de éste análisis emitió cincuenta puntos álgidos relevantes. Entre sus principales conclusiones sobresalen que el número de AM crecerá hasta alcanzar en el 2025 el 10\% de la población.

En México, como en el resto de los países del mundo, los AM cada día son más; actualmente se estima que representan el 5\% de la población. Se calcula que para el año 2030 esta proporción será del 12.6\%5 . La esperanza de vida se ha incrementado, no obstante, buena parte de los últimos años de los AM los viven con padecimientos crónicos, degenerativos, neoplásicos, mentales o infecciosos que limitan de importante manera su calidad de vida. "La morbilidad de los AM se ha incrementado; en
1990 se registraron 479,521 diagnósticos, de una población de 3,259,711 AM lo que corresponde a una tasa de 14, 710 por 100,000 habitantes AM. Estos mismos datos para el año 2005 se elevaron a 2,246,854 diagnósticos, de una población de 5,626,448 y una tasa de 38,970 por $100,000 \mathrm{AM}^{6 \prime}$.

La manera como se ha definido el concepto de salud se ha ido puntualizando, desde que en 1948, la Organización Mundial de la Salud (OMS) definió la salud como el completo bienestar físico, mental y social y no sólo la ausencia de dolencias o enfermedades; este término ha evolucionado desde una definición conceptual hasta una serie de escalas que permiten medir la percepción general de salud. Tanto la vitalidad como el dolor y la discapacidad, se dejan influir por las experiencias personales y por la expectativa de una persona ${ }^{7}$. En la redefinición del concepto de salud, ahora se incluyen las variables de expectativas de salud, capacidad de adaptación al medio, soporte social, autoestima y la habilidad del individuo para competir con limitaciones y discapacidad, mismos que en su conjunto son un indicador de la $\mathrm{CV}^{8}$.

La CV debe de ser entendida como el estado biológico, psicológico, social y económico en el que se encuentra una persona. Desde el punto de vista integral, en su valoración intervienen necesariamente diversos aspectos como la propia experiencia, las creencias, las actitudes y perspectivas que las personas generan con relación a su vida.

La definición de CV tiene componentes subjetivos (el bienestar y la satisfacción) así como componentes objetivos (la presencia de síntomas, el funcionamiento físico y el apoyo social). Diversos autores han coincidido en que cualquier definición que se haga de CV deberá de incluir seis dimensiones: 1) bienestar global, 2) aspectos físicos, 3) psicológico, 4) social, 5) funcional y 6) económico. En síntesis, podría decirse que la CV es la percepción subjetiva del individuo con relación a su incapacidad incluyendo los aspectos de la vida y de la función humana, considerados esenciales para la vida plena?

La CV se basa en la autopercepción de la persona; por consiguiente, los componentes subjetivos son muchos. Aspectos tales como vitalidad, satisfacción, logros y salud son cuestiones en las que la objetividad es difícil sobre todo cuando se utiliza como fuente la autopercepción. En esta investigación se incluyó para la medición de la CV los siguientes aspectos: 1) bienestar global, 2) aspectos físicos, 3) psicológicos, 4) sociales, 5) funcionales y 6) económicos. 


\section{METODOLOGÍA}

Diseño: Estudio observacional, descriptivo y transversal 10,11 que se realizó mediante la aplicación de la "Encuesta para evaluar percepción de la calidad de la atención de Enfermería según el nivel de calidad de vida en los AM" México, $2006 "$.

La población de este estudio consistió en AM que demandaron atención en el Hospital General de México, Clínica 32 del IMSS y Hospital de Xoco, así como en sus domicilios en distintos puntos de la Cd. de México. La técnica de muestreo fue no probabilístico por conveniencia.

\section{Los criterios de inclusión fueron:}

a) AM seleccionados en sus domicilios o que demanden algún servicio de salud en cualquiera de las unidades médicas participantes, b) AM en condiciones de salud física y mental que les permitan uso de razón suficiente para contestar la entrevista de manera lógica y coherente y c) AM que aceptaran participar.

Los criterios de exclusión fueron:

a) Personas que no sean AM

b) AM en estado de coma o con intensos estados alterados de la conciencia

c) AM que no aceptaron participar

Las variables que se estudiaron fueron:

a) sociodemográficas,

b) de vivienda,

c) de salud,

d) demanda de servicios de salud y

e) La CAE, que se evaluó en una escala cualitativa de cinco posibles respuestas, las cuales incluyeron:

1. Cortesía

2. participación de la Enfermera durante el diagnóstico y tratamiento

3. Respeto a la idiosincrasia

4. Trato amable

5. Información sobre el padecimiento,

6. Educación para el cuidado

7. Intimidad y pudor

8. Seguridad y confianza

9. Satisfacción con el equipo y las instalaciones

10. Evaluación general de la calidad de los servicios de Enfermería.

Las 16 preguntas cerradas que se responden de forma cualitativa, tuvieron los siguientes posibles tipos de respuesta: Siempre, Casi siempre, La mitad de las veces,
Casi nunca y Nunca; o bien, Muy buena, Buena, Regular, Mala o Muy mala; y por último Muy bueno, Bueno, Regular, Malo o Muy malo. Posteriormente, se realizó el procedimiento de asignación de valores, se estableció un valor numérico de 10 puntos máximo y de 2 puntos mínimo para cada posible respuesta; la escala de medición quedó de la siguiente manera Siempre (10 puntos), Casi siempre (8 puntos), La mitad de las veces (6 puntos), Casi nunca (4 puntos) y Nunca (2 puntos). Consecutivamente, la calidad interpersonal se agrupó en 10 categorías, de acuerdo al número de preguntas, incluidas en cada una de ellas. La posible puntuación quedó de la siguiente manera: cortesía 30 puntos, participación de Enfermería durante el diagnóstico y tratamiento 20 puntos, respeto a la idiosincrasia 10 puntos, trato amable 30 puntos, información sobre el padecimiento 20 puntos, educación para el cuidado 10 puntos, intimidad y pudor 10 puntos, seguridad y confianza 10 puntos, satisfacción con el equipo y las instalaciones 10 puntos y evaluación general de la calidad de Enfermería 10 puntos, de tal forma que la calificación máxima posible fue de 160 puntos.

La cuantificación de la CV de los AM se midió a partir de los siguientes aspectos: situación de salud, autopercepción del estado de salud, variables exploradoras sobre la capacidad del AM para realizar actividades básicas de autosuficiencia, autopercepción de su estado emocional, autopercepción de su estado de integración social, satisfacción con los logros obtenidos e ingreso económico.

El procesamiento de la información se hizo en dos fases, en la primera de ellas se evaluó la CAE y en la segunda se midió el nivel de CV de los AM. Los datos se analizaron en el programa estadístico SPSS 11.0.

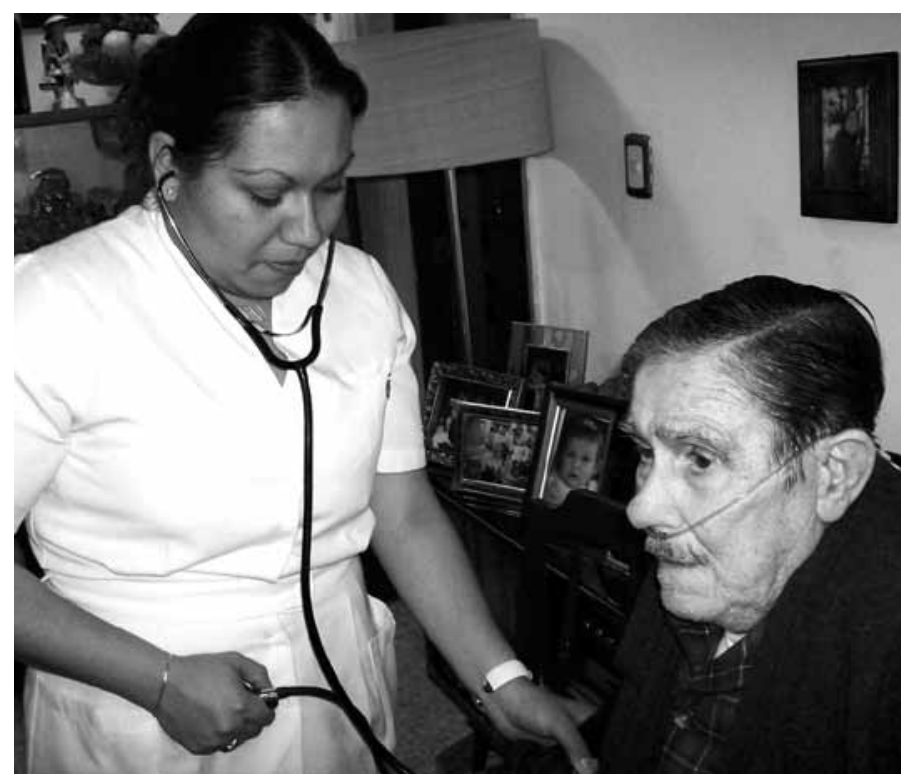


Esta investigación se realizó siguiendo los principios éticos de la "Declaración de Helsinki" "12s, así como las disposiciones que se incluyen en "La ley General de Salud"13.

\section{RESULTADOS}

Se entrevistaron a 82 AM, 37 de ellos en el Hospital General de México, 17 en la Clínica 32 del IMSS y un número igual en el Hospital de Xoco y 11 más en sus domicilios. La distribución por sexo fue la siguiente: 52 mujeres (63.4\%) y 30 hombres (36.6\%), la media de edad fue de 71.6 años y la desviación estándar de 9.1 años.

La escolaridad de los AM fue la siguiente, entre 4 y 6 años de estudio 30 (36.6\%), 20 AM cursaron de 1 a 3 años, otros 14 AM (17.1\%) no cursaron ningún año, el resto de los AM 18 (21.9\%) cursaron más de 6 años de estudio.

Referente a la ocupación actual o última de los AM, 40 (48.8\%) están dedicados al hogar, 33 (40.2\%) se ocuparon en actividades de servicios públicos y personales tales como obreros, chóferes, albañiles, empleados, etc. Otros 5 (6.1\%) AM a otras ocupaciones y únicamente 4 (4.9\%) fueron profesionistas.

Declararon poseer vivienda propia 54 (65.9\%) de los AM, rentada $18(22.2 \%)$ y prestada $9(11.0 \%)$. En cuanto al número de dormitorios por vivienda, 18 (22.0\%) contestaron que en sus viviendas sólo existe un dormitorio, hasta 2 dormitorios 33 viviendas (40.2\%), hasta 3 dormitorios 18 viviendas (22.0\%) y 4 y más dormitorios 16 (19.5\%). Aproximadamente el 90\% de las viviendas de los AM son de materiales de construcción de tipo permanente y cuentan con servicios básicos, solamente 60 (73.3\%) viviendas cuentan con baño tipo inglés y regadera.

La situación de salud de los AM fue la siguiente, 40 (48.8\%) tenían diagnóstico médico de enfermedad crónico-degenerativa, principalmente diabetes mellitus e hipertensión arterial; 13 (15.8\%) presentaban algún tipo de neoplasia; las enfermedades mentales y las discapacidades estuvieron presentes en 12 AM (14.6\%); las infecciones respiratorias y diarreicas en 7 (5.8\%) AM y el dolor crónico principalmente asociado a fracturas o artritis en 6 (7.3\%) AM.

En general la calificación que asignaron a la CAE para trato amable, respeto, enseñanza del cuidado y satisfacción con las instalaciones fue considerada como "Muy Buena" o "Buena" en $80 \%$, sin embargo se observa una marcada tendencia de acuerdo al sexo femenino por asignar calificaciones más altas para los rubros de "Buena y Muy buena" (Ver cuadro 1).

\section{Cuadro 1}

\section{Calificación en general de la CAE por sexo}

\begin{tabular}{|l|c|c|c|}
\hline \multirow{2}{*}{$\begin{array}{c}\text { Calidad } \\
\text { CAE }\end{array}$} & \multicolumn{2}{|c|}{ Sexo } & \multirow{2}{*}{ Total } \\
\cline { 2 - 3 } & Masculino & Femenino & \\
\hline Muy buena & $7(28 \%)$ & $18(72 \%)$ & $25(31 \%)$ \\
\hline Buena & $13(32 \%)$ & $28(68 \%)$ & $41(51 \%)$ \\
\hline Regular & $6(55 \%)$ & $5(45 \%)$ & $11(14 \%)$ \\
\hline Mala & $2(100 \%)$ & 0 & $2(3 \%)$ \\
\hline Muy mala & $1(100 \%)$ & 0 & $1(1 \%)$ \\
\hline Total & $29(36 \%)$ & $51(64 \%)$ & $80(100 \%)$ \\
\hline
\end{tabular}

La CV se midió en una escala de 100 puntos; se asignaron 10 puntos para cada uno de los siguientes rubros: vivienda, situación de salud, estado emocional, integración social y familiar, satisfacción con los logros obtenidos y ingreso económico; se calificó con 20 puntos la percepción del estado de salud y el estado funcional del individuo.

Los resultados generales de la medición de la CV se muestran en el (cuadro 2).

\section{Cuadro 2}

\section{NIVEL DE CV EN AM}

\begin{tabular}{|l|l|c|c|}
\hline \multicolumn{1}{|c|}{ Puntos } & \multicolumn{1}{|c|}{ Nivel de CV } & $\mathbf{N}^{\circ}$ & \% \\
\hline 0 a 40 & Baja & 2 & 2.4 \\
\hline 41 a 50 & Media baja & 10 & 12.2 \\
\hline 51 a 60 & Media & 20 & 24.4 \\
\hline 61 a 80 & Media alta & 40 & 48.8 \\
\hline 81 a 100 & Alta & 10 & 12.2 \\
\hline Total & & 82 & 100 \\
\hline
\end{tabular}

El análisis de la correlación en general fue baja. La correlación de Pearson entre la CV y CAE fue de .358, esta se incrementa a .566 cuando se analizan por separado los AM del Hospital de Xoco. Al realizar el cruce de CV, CAE y percepción del estado de salud, la correlación es de tan sólo .331, las más altas correlaciones se obtuvieron al cruzar CV y años de estudio .435 y CV contra función física. 


\section{DISCUSIÓN}

Los estudios sobre CAE generalmente no están considerando a la CV como un modificador de la percepción de la CAE. Cuando los AM han requerido cuidados de Enfermería y posteriormente se les pregunta su opinión acerca de la CAE recibida, su respuesta estará influenciada conforme -al menos- cuatro diferentes situaciones: a) sus creencias, conocimientos y actitudes; b) su nivel de CV; c) la severidad del problema de salud que originó el acto del cuidado y d) las políticas de contratación de personal, ya que, cuando el personal es insuficiente o carece de la preparación profesional necesaria, en lo primero que se vera reflejada esta carencia será en un déficit de CAE.

Por consiguiente, es muy importante que también se tomen en cuenta el nivel de CV al realizar estudios de medición de la $C A E$, ya que la CV tiene un efecto modificador en la objetividad de la opinión de los AM en cuanto a la CAE.

Si bien los resultados del análisis de correlación fueron poco satisfactorios, esto no necesariamente indica que la CV no sea un elemento que modifica la percepción de la CAE. Otro aspecto a considerar es el número de AM encuestados y la técnica de muestreo, así como la dependencia del cuidado, ya que a muchos de ellos se les entrevistó en servicios hospitalarios que demandan poco cuidado de Enfermería.

\section{CONCLUSIONES}

Las mujeres tienen una opinión más favorable de la $C A E$, no así en los hombres en donde las opiniones desfavorables se incrementan conforme a la edad, al bajo nivel educativo, la pobreza y la severidad del diagnóstico.

Las opiniones favorables sobre la CAE están relacionadas con la mayor necesidad de cuidado y niveles de CV medios y altos.

La correlación de Pearson entre la CV y CAE fue baja (.358), pero cuando se seleccionó únicamente a los AM atendidos en el hospital de Xoco, quienes consideramos eran de los que tenían mayor necesidad de cuidado, ésta se eleva a .566. Las asociaciones que mejor correlacionaron fueron CV y años de estudio (.435) lo que indica que probablemente el nivel de educación influya en la percepción de la CAE.

En el caso de la CV contra función física, que de alguna manera podría ser un indicador de la necesidad de cuidado, la correlación fue de .505 .

Finalmente, se requiere de continuar trabajando esta línea de investigación para poder determinar cuáles son los factores presentes en la población de AM que modifican su nivel de percepción y aumentan el nivel de subjetividad en la medición de la CAE.

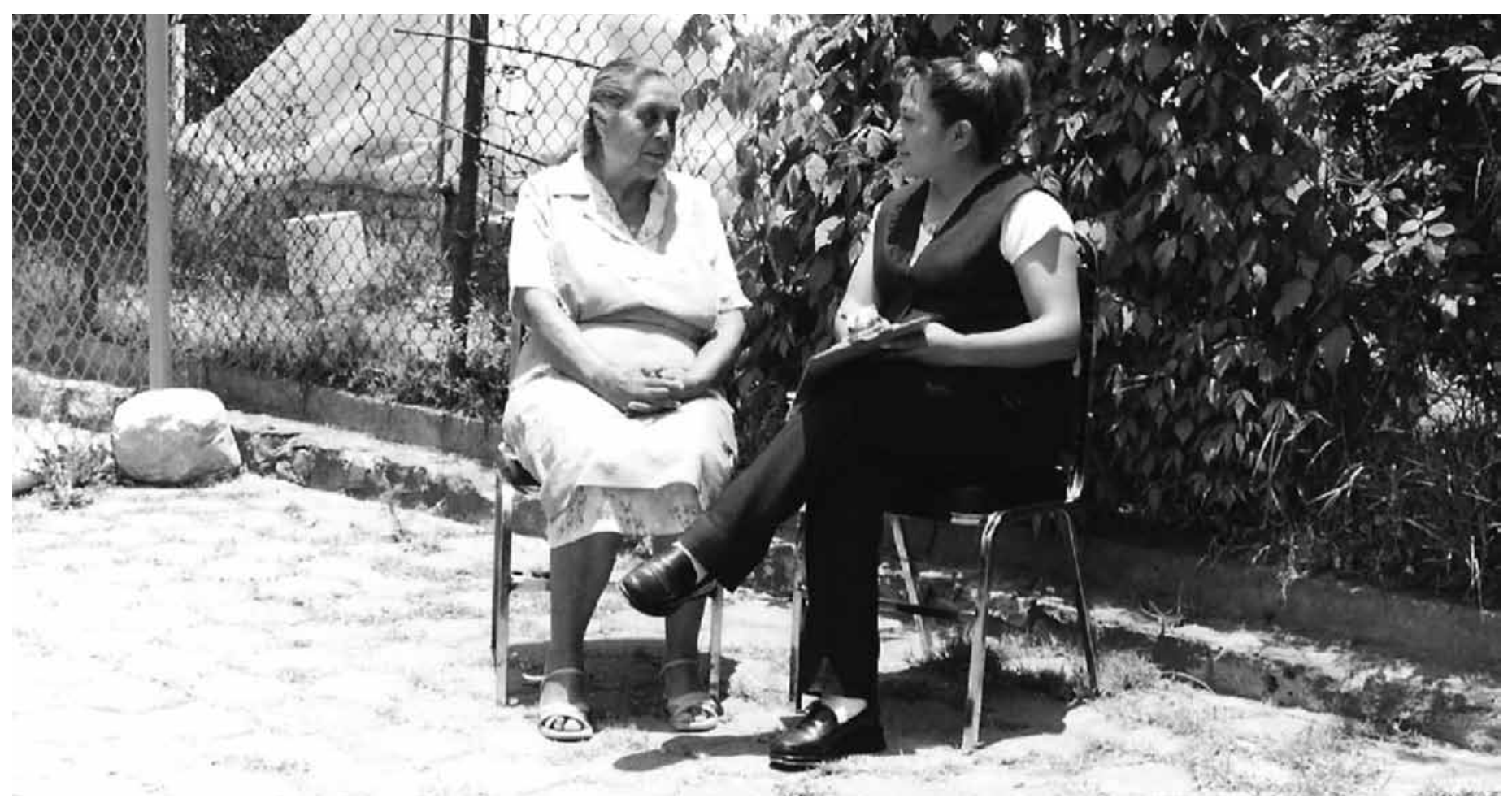




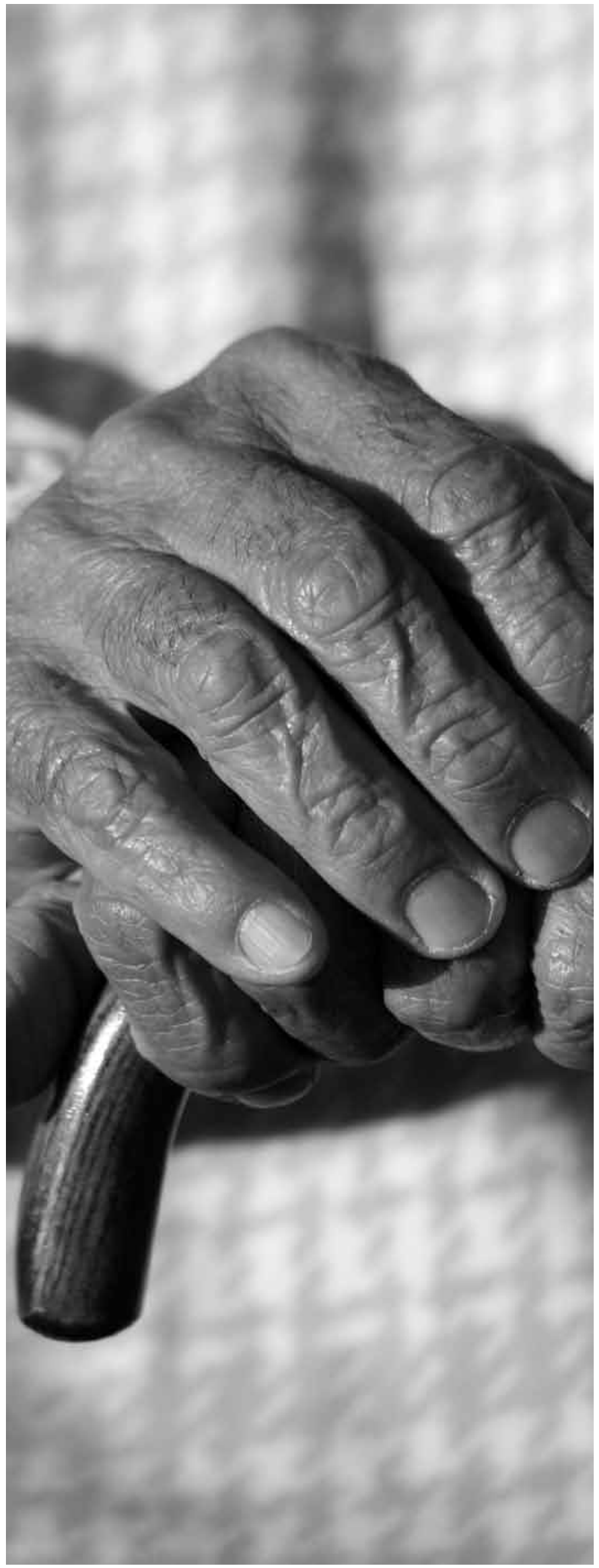

\section{REFERENCIAS BIBLIOGRAFICAS}

1 Secretaría de Salud, Programa Nacional de Salud 2000-2006. México, 2000. pp 52-56.

2 Secretaría de Salud. Comisión Interinstitucional de Enfermería. Evaluación de la calidad de los servicios de Enfermería: tres indicadores de aplicación hospitalaria. México, 2003. pp 36-45.

3 Ortega Vargas C, Suárez Vázquez MG, Jiménez y Villegas MC, Añorve Gallardo A, Cruz Corchado M, Cruz Ayala G, Quintero Barrios MM, Solís Pérez MT y Zárate Grajales RM. Manual de evaluación del servicio de calidad de Enfermería: estrategias para su aplicación. $1^{\text {a }}$ edición. Editorial médica panamericana. México. 2006. p 8.

4 Organización Mundial de la Salud. Cincuenta Puntos del Informe Mundial en Salud, Situación Global y Tendencias 19552025. Ginebra Suiza, 1998. pp 1-4

5 Negrete Salas ME. Distribución geográfica de la población mayor. Demos. Instituto de Investigaciones Sociales UNAM. 2001, México. pp 18-20.

6 Molina Sánchez MR. Panorama epidemiológico del adulto mayor en el 2005. Epidemiología. Secretaría de Salud. Dirección General de Epidemiología. México. Partes 1 y 2. 2006; 23: $(8,9)$ pp 1-3.

7 Pineault R, Daveluy C. La planificación sanitaria: conceptos métodos estrategias. Capítulo 1. Ed. Masson. Barcelona, España 1995. pp 1-41

8 Valverde Jurado E, Ávila Figueroa C. Evaluación de la calidad de vida. Salud Pub Mex. 2002; 44: 349-3.

9 Lara Muñoz MC, Ponce de León S, de la Fuente JR. Conceptualización y medición de la calidad de vida de pacientes con cáncer. Rev Invest Clin. 1995;47; 315-27

10 Dos Santos-Silva I. Estudios transversales. En: Dos Santos-Silva I. Epidemiología del cáncer: principios y métodos. Lyon, (Francia): Agencia Internacional de Investigación sobre el Cáncer/ Organización Mundial de la Salud, 1999: 225-244.

11 Hernández Ávila M, Garrido Latorre F, López Moreno S. Diseño de estudios epidemiológicos. Sal Pub Mex. 2000; 42: 144154.

12 Ramírez Rivera N A. Declaración de Helsinki sobre ética en la investigación médica. Rev Méd UV. 2002; 2(1): 5-9.

13 Secretaría de Salud. Ley General de Salud. México, 2003. pp. 20-22.

\section{DIRECCIÓN PARA CORRESPONDENCIA}

Rey Arturo Salcedo Álvarez: rasalced@hotmail.com 